\title{
Opportunities and challenges of the Internet of Things for healthcare
}

\author{
Systems engineering perspective
}

\author{
Felipe Fernandez \\ Department TFB-ATSI FI-ETSIINF \\ Polytechical University of Madrid (UPM) \\ Campus de Montegancedo, 28660 Madrid, Spain \\ felipe.fernandez@es.bosch.com
}

\author{
George C. Pallis \\ T4i Engineering Ltd \\ 40 Gracechurch Str. EC3V 0BT \\ London, United Kingdom \\ gpallis@t4i.co.uk
}

\begin{abstract}
In the incoming world of Internet of Things (IoT) for healthcare, different distributed devices will gather, analyze and communicate real time medical information to open, private or hybrid clouds, making it possible to collect, store and analyze big data streams in several new forms, and activate context dependent alarms. This innovative data acquisition paradigm allows continuous and ubiquitous medical data access from any connected device over the Internet, and a novel health application ecosystem emerges. In these complex ecosystems could be insufficient to discuss only classical requirements regarding hardware issues and software support of individual elements. In the involved multidisciplinary development area, with intricate vertical and horizontal markets, it is essential a close collaboration between the corresponding stakeholders: endusers, application domain experts, hardware designers, software developers, market specialists, road mapping strategists and even the collaboration of visionaries to implement successful healthcare ecosystems. In this paper we describe some crucial systems engineering viewpoints to analyse the corresponding complex decision space. We complement the general examination of the IoT space by commenting some particular examples and specific details, which correspond to remarkable options of the involved dimensions.
\end{abstract}

Keywords: Internet of Things (IoT), systems engineering, healthcare, eHealth, mHealth, medical body area networks, big data analytics, cloud computing.

\section{INTRODUCTION}

Healthcare organizations around the world are transforming themselves into more efficient, coordinated and user-centered systems. This tendency implies more integrated interoperable and ubiquitous healthcare services, a greater and easier access to healthrecords and related information, and to engage patients in their own healthcare. With this purpose, the information and communication technology should play a more central role in achieving efficiencies and enhancing distributed healthcare systems that should fulfill diverse and frequent update demands. In a little provocative way, one could paraphrase George Clemenceau (1841-1929) by saying that perhaps health is too important to be left only to doctors.

To address health issues, the Internet of Things (IoT) offers a world of networked devices, cloud based applications and services, with diverse cooperation mechanisms and where big data analytics enables getting objective data and information, and well-grounded decision making (Figure 1).

Health-IoT ecosystems today are based on a network of devices that connect directly with each other to capture and share vital data, and significant context information, through a secure service layer that connects to a central command and control server in the cloud.

Relevant segments of the home healthcare market are: independent living services, consumer medical devices, telehealth, wearable technologies, monitored fitness devices, health gaming and personal emergency response systems. Particularly, in the field of telecare, remote monitoring of patients allows more self-management of chronic conditions, and significant service improvements and cost reductions.

The future success of IoT mainstream will be based on the confluence of a right standardization, efficient wireless protocols, improved sensors, cheaper and low-power microprocessors, advanced SoC, and the support of communities and established companies for the necessary development of cloud-based applications.

It is expected that by 2020 the number of internet connected devices will likely reach 50 billion, and the increased traffic from those devices will probably have a substantial effect on networks, data centers and smart connected products.

The general interrelated applications that appear to be prime for IoT disruption include remote monitoring, home automation, appliances of all types, wearable devices, home and hospital health care, retail and warehousing inventory management, connected farms equipment, and likely many more that have not yet been invented.

IoT phenomenon can also play a central role in achieving efficiencies and improvements to meet diverse healthcare demands. Medical centres, hospitals, physicians and associated systems must facilitate the reliable flow of patient data throughout an expanding community of care, while also securing the information and rigorously protecting patient privacy. To accomplish this, healthcare IT platforms must be as flexible and rapidly scalable as they are highly secure. 
Health IoT ecosystems has to deliver the right information, at the right time, to all key stakeholders, in order to have a real Information-driven Healthcare.

The systems engineering (SE) and system-of-systems engineering (SoSE) viewpoints [1] ensure that the elements of Health-IoT ecosystems fit together to achieve the general objectives of the corresponding stakeholders, and fulfill the associated evolving programs.

This paper analyses the involved system engineering decisions, to build cost-effective Health-IoT platforms that enhance the corresponding medical services, clinical care and remote monitoring, to respond to new society challenges.

\section{SYSTEM ENGINEERING DECISION SPACE}

A decision space, in system engineering, is usually defined by a set of dimensions and range of options at the decision maker's disposal. For each dimension there is a set of constraints and derived possible consequences. In this paper, we consider the associated system engineering viewpoints to practically explore the corresponding Health-IoT ecosystems. And for decision-making it is advisable to remember that to arrive at the optimal solution, it's not always easy.

In this paper, we only enclose some crucial dimensions (12) that usually have a significant impact on the involved HealthIoT ecosystems. The associated dynamical space should be regularly analyzed for controlling the strategy of considered healthcare developments. Where appropriate, we analyse some particular examples of the corresponding dimension to illustrate the involved factors.

\section{A. Business model}

It is usually recognized that historically, breakthrough technologies needed innovative business models to become successful. Relevant factors in this dimension are: context, vision, mission, SWOT (strengths, weaknesses, opportunities, and threats) analysis, value chain, consumerization, marketability, revenue streams, market segments, distribution channels, partnership, strategic alliances, key resources and process, cost structure, and ecosystem laws.

Investigating the corresponding vertical and horizontal markets is crucial when deciding on the IoT business model, product scope, growth method, long-term strategy, and for moving either vertically or horizontally, and for choosing the right direction. Typically it is better to move in one direction at a time.

Some technical developers are contentedly tinkering with Arduino or Raspberry Pi modules, clip cables, IDLE Python, pluggings and demonstrating cool applications. But IoT system engineering requires also consumer focused and formal analyses of the application scope, business integration, synergies, scale economies, suitable resources, roadmaps, interoperability, standardization and deep investigation of the market; in order to achieve the high potential of IoT ecosystems. IoT should be used as an enabling technology for remote health monitoring, which allows building cloud hub platforms, to capture patient data from a variety of sensors and securely stores the corresponding information in the cloud.

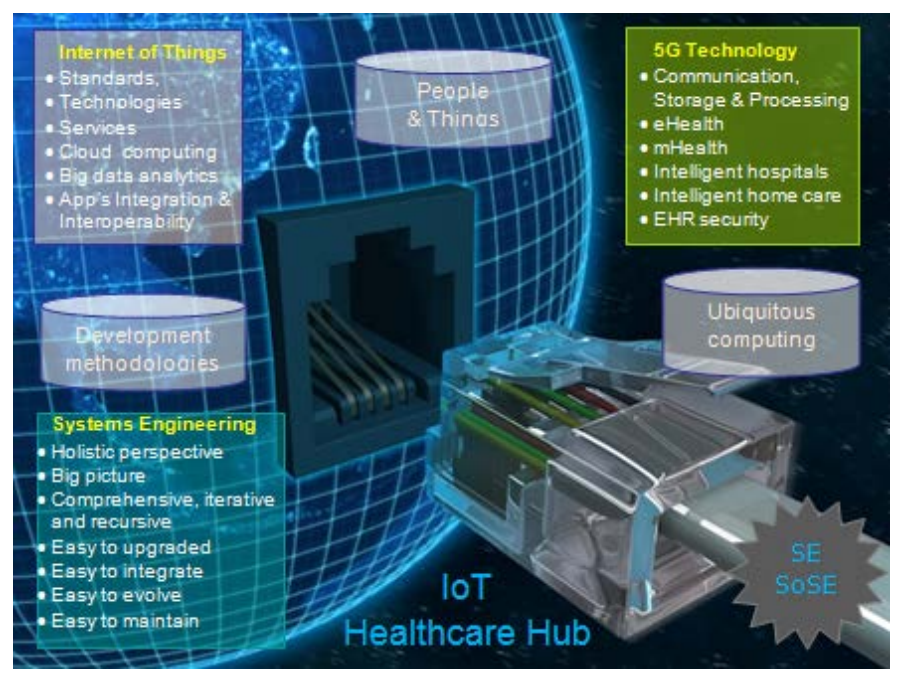

Fig. 1. The Internet of Things as a global hub for Healthcare

The fundamental stimulus of IoT should come from constructing intelligent objects that contain sensors, controllers, software and possibly user interfaces, connected to different cloud services. It is the combination of data, analytics and intelligence coupled with worldwide connectivity and location that gives the great potentiality to IoT business models.

\section{B. Nonfunctional requirements}

In general, nonfunctional requirements or system qualities describe system attributes or constraints. They are often referred as the "ilities". They can also be restrictions on the design of the involved system. For IoT systems for healthcare applications, the following nonfunctional requirements are commonly critical: safety, security, privacy, reliability, robustness, stability, availability, resilience, usability, accessibility, scalability, interoperability, flexibility, extensibility, modifiability, testability, portability, maintainability, power efficiency and QoS. Open source and open platform requirements are in many cases advisable.

Nonfunctional requirements are just as critical as the functional features and user stories, as they assure the usability and effectiveness of the entire IoT ecosystem; failing to meet any one can result in systems that do not meet end-user, market or production needs, or requirements that are imposed by regulatory or standards agencies. The ilities priority specification of the considered healthcare application is relevant to guide the corresponding IoT system design.

For suitable IoT deployments, it is usually recognize the necessity of five S's that are key to the growth of this ecosystem: Simplicity, Staying Power, Stability, Security and Standards. Additionally it is also considered important the necessity of having Smart characteristics like: automated remote management and configuration, multimodal HMI's, awareness of context and location, multimodal communication, local processing and analysis capabilities, automated monitoring and control, possibility to anticipate and predict critical events, adaptive behavior and also attractive. Smart healthcare products should also have a pleasant look and feel. 
Smart IoT systems, based on the above characteristics, will help accelerate the rate of IoT adoption in health areas.

\section{Application context and physical environment}

The deployment of healthcare wireless sensors for concrete physical environments and specific applications may take several ways and implies different constraints. The type of environment and application determine important properties of the involved sensors and networks, such as necessary availability (MTBF), redundancy, reliability and robustness, or suitable spectrum frequencies, antenna range and density or power supply constraints. For example, healthcare applications and devices derived from common smartphones phones cannot be suitable for critical healthcare applications that require high availability or long periods of working.

\section{Diagnosis and monitoring health sensors}

Present wearable health devices include heart rate monitors, ECG monitors, glucose monitors, pulse oximeters and blood pressure monitors. In the near future, they are expected to be complemented with micro and nano chemical sensors that will provide continuous medical diagnostics. These miniaturized smart sensors will be able to detect additional chemical signatures, e.g. in breath and sweat, which can be translated into medical monitoring (e.g. diabetes patients, other metabolic diseases, skin diseases and drug pharmacokinetics).

\section{E. Node physical characteristics}

The involved sensor and devices may require different characteristics: form factor, size, weight, power consumption; mobility and ergonomic requirements; IP \& IK protection degrees, battery lifetime; connectivity; processing and positioning requirements; multimodality and communication range; throughput and latency constraints; self-healing and EMC requirements; cost constraints, etc., which can be crucial for the corresponding healthcare IoT ecosystem.

\section{F. Market analysis and positioning}

The healthcare IoT market includes the following segments: hospital health services, home health services, independent living services, consumer medical devices, telehealth, personal emergency response systems, health and fitness monitoring and wearable technologies. The suitable selection of the IoT market segment, positioning, and target customer are essential for the success of the corresponding business model. IoT healthcare technology vendors are developing highly connected and interoperable solutions taking advantage of patient-generated data to support the decision-making process of healthcare professionals, and to create the inevitable bridge between clinical care and home health.

Wearable health technologies should play an important role in the continuum of care and in the concept of constantly managing patients' health to attain higher quality of health services and lower healthcare expenditures. Meanwhile, the quantity of patient-generated data will grow exponentially with the increasing use of wearable health technologies and big data services. A mayor requirement for the rise of home health technologies is convergence. From a technology standpoint, the market will experience different convergences in order to obtain more efficient health systems and services [2].

\section{G. Portfolio of wireless networks}

Common wireless technologies are based on IEEE standards [3]. The most relevant for sensor networks are for LANs: Wi-Fi $2.40 \mathrm{GHz} \& 5 \mathrm{GHz}$ (IEEE $802 \mathrm{n}$ ac); and lowpower 900MHz (IEEE 802.110ah); ZigBee \& ZigBee PRO 2.4 GHz \& $900 \mathrm{MHz}$ (IEEE 802.15.4) and 6LoWPAN (for IoT); for PANs: Bluetooth $2.4 \mathrm{GHz}$ (IEEE 802.15.1) and Bluetooth low energy (BLE); UWB (IEEE 802.15.4a); RFID (IEEE 802.15.4f) and Low Rate WPAN (IEEE 802.15.6), which are the IEEE standards associated with the body area network (BAN). For very low-power: DASH7 based on $433 \mathrm{MHz}$ (ISO/IEC 18000-7). Presents standards for mobile cellular networks include: GSM / GPRS / UMTS / HSPA / LTE. Proprietary wireless networks still play something of a role in healthcare environments in general and IoT applications in particular, but that role seems to be shrinking as the industry continues to move toward standards-based architectures.

The French company SIGFOX [4] has developed a specific radio communication technology to increase the effective bandwidth, by diverting IoT traffic from the Internet through a special ultra low-bandwidth and very low energy consumption cellular network (900 MHz) to support IoT connections. San Francisco will be the first U.S. city to deploy the SIGFOX IoT network. Whether a separate IoT network is needed, however, is debatable: the three big available wireless technologies (cellular wide area networks, Wi-Fi local area networks and Bluetooth Smart personal area networks) are perhaps sufficient for common IoT networks.

\section{H. The fifth generation of communication networks}

The 5th communication technology for people and things (Internet of Everything) will be a truly converged network where wired and wireless communications will use the same infrastructure. This future ubiquitous, ultra-high bandwidth communication infrastructure will drive the future networked society [5]. The 5G communication technologies are expected to allow: 100 times higher wireless bandwidth compared to 2010; up to $90 \%$ of energy saving per service provided; high optimization and variety of delivered services for communication, storage and processing, and big data analytics; very dense deployments of wireless communication links to connect over 7 trillion wireless devices serving over 7 billion people; and to enable advanced user controlled privacy.

\section{Medical body area networks}

Medical Body Area Networks (MBAN's) open the door for monitoring systems — which currently attach patients to machines using a mass of wires - to operate wirelessly using low-cost wearable sensors. Wired monitoring systems make it difficult for patients to move about, and they increase the chances for errors and hospital-acquired infections.

MBAN's enable hospitals to monitor a much larger percentage of patient population and more quickly identify health events that require intervention. This, in turn, should lead to better patient care, improved outcomes and lower costs. 
For example, a monitored hospital patient would have a roughly $48 \%$ chance of surviving a cardiac arrest, compared with a $6 \%$ chance for unmonitored patients.

FCC has approved a specific spectrum for wireless networks, used to monitor patient data within healthcare facility MBAN devices, in the 2360-2400 MHz band. The decision to open the use of this frequency range is part of an effort to reduce the chances that other wireless devices such as mobile phones could interfere with MBAN transmission [6].

Examples: Verizon, Philips, Qualcomm and GE are developing MBAN devices for clinical diagnoses to deliver better patient care at lower costs. Which offer interoperable solutions to remotely monitor patients: glucose levels, weight, heart rate, and blood pressure at their homes, and send the real time biometric information to healthcare providers onto PCs, tablets and smartphones. They also provide modules for remote monitoring and analysis of sport-fitness activities.

\section{J. Personal health device communication standards}

Common reference models for making personal health data available in transport-independent transfer syntax enable to establish logical connections between systems and to provide communication services. The protocols should be optimized to personal healthcare requirements and commonly used methods and tools. Example: ISO/IEEE 11703-20601:2010 exchange protocol for health informatics and personal health device communication [7].

\section{K. Wearable technology and cloud platforms}

Real time data and context information provided by wearable medical devices with associated electronic health records can be extremely valuable assets for caregivers and a significant source for scientists, since they have the possibility to easily capture continuous patient data and context information outside the facility walls. This can be accomplished by prescribing medical devices that can monitor and securely store health information to a central repository or cloud, which provide continuous flows of information for extended periods of time, in order to achieve real predictive healthcare and also for detecting possible unhealthy habits.

Twelve concrete examples of cloud-based platforms for IoT developments are: Thread, OIC, AllSeen, HyperCat, IIoT, IIC, Xively, HomeKit, HealthKit, Withings, Fitbit and Epic-IBMApple. Many medical device manufacturers are looking to integrated advanced sensor technologies and suitable cloud platforms for continuously listening to our bodies, analysing the signals and contexts, and continuous healthcare services.

A particular ecosystem from Samsung Electronics is the Simband and Samsung Architecture for Multimodal Interactions (SAMI) platform. This is a reference ecosystem for personal health devices with wearable wristband form factor, based on an open and modular hardware-software architecture and a flexible cloud-based software platform, for the community development of advanced sensors and algorithms, as well as data collection and analysis. These devices with multimodal HMI's could be used to track and display measurements such as heart rate, respiratory rate and blood pressure. Additionally, they are capable of integrating advanced new sensing technologies [8].

\section{Big data analytics technologies}

Connectivity and cloud computing supply the critical building blocks for information-driven and patient-centered healthcare systems. They should provide the flexibility and scalability that enables hospitals and medical centers to be more agile for the constant change, with the aid of cloud technology, to speed the corresponding transformations. The involved big data analytics (commonly associated with five V's: volume, variety, velocity, veracity, and value) should allow: data storing, reporting, analysis, visualization, monitoring, prediction; and decision-making, which are crucial for the continuous improvement of healthcare services.

New communication technologies like Software Defined Networking (SDN) and Software Design Data Centres (SDDC) are being developed to make it easier to provision and manage networks as they grow to support the massive increases in Internet bandwidth and complexity the IoT brings. Many of the concepts of the IoT not only drive increases in bandwidth and device counts, but also drive requirements for lower latency, greater determinism and processing closer to the edge of the network (called Fog Computing) to reduce latencies and network bandwidth requirements. Support for these IoT concepts are being driven into next generation routers, switches and networking software.

\section{CONCLUSIONS}

In IoT space there are usually many different technical alternatives for healthcare applications, which means that the concrete projects should analyze the whole set of main possible solutions in order to determine the optimal ones, considering the particular constrains and priorities of the corresponding applications. Therefore, it is important to have a structured systems engineering methodology to guide the corresponding decision processes for developing Health-IoT ecosystems.

\section{References}

[1] MITRE's Systems Engineering Guide. www.mitre.org

[2] IHS Medical Devices \& Healthcare IT, Home Health Technology Report - 2014. www.ihs.com

[3] IEEE 802 WPAN/LAN/MAN Standards www.ieee802.org

[4] SIGFOX radio connectivity solution www.sigfox.com

[5] EC Horizon 2020. Digital Agenda for Europe. Towards 5G. http://ec.europa.eu/digital-agenda/en/towards-5g

[6] MBAN, Medical Body Area Networks, FCC 12-54, ET Docket No. 0859, Released: May 24, 2012, Federal Communications Commission, www.fcc.gov/document/medical-body-area-networks

[7] ISO/IEEE 11073-20601:2010 Health informatics -- Personal health device communication -- Part 20601: Application profile -- Optimized exchange protocol. www.iso.org

[8] Samsung's Simband platform, Digital Health Innovation Lab, http://www.samsung.com/us/globalinnovation/innovation_area 